\title{
Rectosigmoid Colon
}

National Cancer Institute

\section{Source}

National Cancer Institute. Rectosigmoid Colon. NCI Thesaurus. Code C103438.

A portion of the large intestine that includes the descending colon, sigmoid colon and rectum. 